\title{
Electrically doped SiGe-heterojunction TFET based biosensor considering non-ideal hybridization issues: a simulation study
}

\author{
Basudha Dewan ${ }^{1} \cdot$ Shalini Chaudhary ${ }^{1} \cdot$ Menka Yadav $^{1}$
}

Received: 18 July 2021 / Accepted: 14 September 2021 / Published online: 25 September 2021

(c) The Author(s), under exclusive licence to Springer-Verlag GmbH, DE part of Springer Nature 2021

\begin{abstract}
Calibrated simulations are used to study a dielectric modulated, electrically doped, dual metal gate, SiGe heterojunction, double gate TFET biosensor in this work. Use of lower bandgap SiGe at the source side helps in improving the ON current of the biosensor. Electric doping is preferred over physical doping to overcome the random dopant fluctuations and high thermal budget problems. Non-ideal situations having partially and non-uniformly filled cavity regions are analyzed in this work. Partially filled cavity has fill factor less than $100 \%$ and is studied by considering $50 \%, 20 \%$, and $10 \%$ fill factors. Different positions of biomolecules inside a partially filled cavity are also studied through extensive simulations and are found to affect the sensitivity largely. Four different non-uniform profiles, decreasing, increasing, convex and concave, are created in the cavity region and their sensitivity values are compared for different dielectric constants $(k)$ and charge densities $\left(Q_{\text {Bio }}\right)$. Among the different non-uniform profiles considered, maximum sensitivity is obtained for decreasing profile and it improves with an increase in dielectric constant and positive charge density while it decreases when negative charge density increases.
\end{abstract}

Keywords TFET · Dielectric modulation - Embedded cavity · Electric doping · Threshold voltage · Sensitivity.

\section{Introduction}

Research interest in the field of biosensors has gained a lot of attention because of the increased threat posed by bio-attacks to the mankind. COVID-19 is the recent example of the bio hazard which has affected the entire world. It becomes very important to detect the presence of biomolecules that are around us to avoid any possible health hazards [1]. Biosensors utilized for this detection process detect a particular biomolecule and create an electrical signal corresponding to it [2]. Biosensors nowadays are utilized in various fields like medical diagnosis, food processing industry, pollution level detectors, agricultural industry, etc [3]. Researchers are working towards creating highly sensitive, reliable, robust, and cost-effective biosensors for the benefit of society. The first enzyme-based biosensor was discovered by Clark et al. in 1962 [4].

Basudha Dewan

2019rec9012@mnit.ac.in

1 Department of Electronics and Communication Engineering, Malaviya National Institute of Technology Jaipur, Jaipur, Rajasthan 302017, India
Researchers have commonly used FET-based biosensors for their ability of label-free detection, CMOS compatibility, cost-effectiveness, and miniaturization [5-7]. But the downside of these FET-based biosensors is short channel effects (SCEs) and large subthreshold swings (SS> $60 \mathrm{mV} /$ decade) [8-10]. Tunnel FET (TFET)-based biosensors have been proposed by researchers with improved response time and sensitivity to address these disadvantages. The current is regulated by the band-to-band tunneling (BTBT) mechanism in TFET-based biosensors, which makes them very useful in biosensing applications $[8,11-13]$. However, the problem with TFET is its low ON-state current in comparison with MOSFET and ambipolarity effect. These issues are resolved by using different structural variations such as pocket doping, heterojunction, work-function engineering, bandgap engineering, dielectric modulation (DM) and electric field imbalance. In dielectric modulated TFETs, the cavity created in the gate dielectric region is used for the accumulation of biomolecules. Dielectric constant and charge density of the cavity change upon the accumulation of biomolecules in comparison with empty cavity which has dielectric constant $k=1$ and no charge density. This further leads to change in the threshold voltage, drain current and sensitivity of the biosensor. 
This article proposes an electrically doped, dual metal gate, SiGe-heterojunction, double gate TFET biosensor. Physical doping of TFET is avoided because it makes the fabrication process complex, raises the thermal budget of the entire process, and contributes to random dopant fluctuations (RDFs) [14]. In electrically doped TFET-based biosensor, the source and drain regions are induced using metal electrodes of suitable work-functions. Two conditions that need to be fulfilled in case of the electrically doped TFET are [15] :

1. Thickness of the silicon body must be kept less than Debye's length given as

$$
L_{D}=\sqrt{\frac{\epsilon_{\mathrm{Si}} \times V_{T}}{q \times N}}
$$

where $\epsilon_{\mathrm{Si}}$ is the dielectric constant of silicon, $V_{T}$ is thermal voltage and $N$ is the density of biomolecules in the cavity.

2. Work-function values for source and drain electrode are given as

$$
\begin{gathered}
\Phi_{\text {source }}>\left(\chi_{\mathrm{Si}}+\frac{E_{g}}{2 \times q}\right) \\
\Phi_{\text {drain }}<\left(\chi_{\mathrm{Si}}+\frac{E_{g}}{2 \times q}\right)
\end{gathered}
$$

where $\chi_{\mathrm{Si}}$ is the electron affinity of silicon which is approximately $4.17 \mathrm{eV}$ and $E_{g}$ is the energy bandgap of Silicon.

In our previous work, we have investigated the performance of a fully and uniformly filled cavity, i.e., ideal case. But this may not be the case in practical situations because the receptors or probes immobilize the biomolecules entering the cavity region. The hybridization process can stop before the cavity is completely packed. It prevents the entry of more biomolecules into the cavity region. A condition called steric hindrance is induced. Therefore, extending our previous work, here we have studied the behavior of partially filled cavity having fill factor less than $100 \%$ and different non-uniform profiles in the cavity region. Partially filled cavity has fill factor less than $100 \%$ and is studied by considering $50 \%, 20 \%$, and $10 \%$ fill factors. Different positions of biomolecules inside a partially filled cavity are also studied through extensive simulations and are found to affect the sensitivity largely. Four different non-uniform profiles, decreasing, increasing, convex and concave, are created in the cavity region and their sensitivity values are compared for different dielectric constants $(k)$ and charge densities $\left(Q_{\text {Bio }}\right)$.
The structure of the device along with the simulation setup used is discussed in Sect. 2. The impact of variation in fill factor, position of biomolecules, non-uniform profiles and molar concentration of germanium on the device performance is discussed in Sect. 3 followed by the sensitivity analysis in Sect. 4. The conclusion is drawn in Sect. 5.

\section{Device structure and simulation methodology}

\subsection{Proposed device architecture and working}

The structure of the proposed electrically doped, dual metal gate, SiGe-heterojunction double gate TFET (ED-DMGSiGe-HDG-TFET) biosensor following International Roadmap for Devices and Systems (IRDS) standards [16] is presented in Fig. 1. Table 1 shows the device parameters and the values applied to them during the simulation which are agreeing with the device dimensions given in [17]. Due to its lesser bandgap, $\left.\mathrm{Si}_{(1-x)}\right) \mathrm{Ge}_{(x)}$ having $x=0.5$ is used as the source material which leads to an improved ON current. To induce a $P^{+}$source region, a platinum electrode with a work function of $5.93 \mathrm{eV}$ is used and hafnium electrode with a work function of $3.9 \mathrm{eV}$ is used to induce a $N^{+}$drain region. A thin layer of $\mathrm{SiO} 2$ is deposited between the $\mathrm{SiGe}$ source and the source electrode to prevent silicide formation [18]. To reduce the ambipolar effect, the gate work function is kept higher on the drain side, i.e., $\Phi_{M 2}>\Phi_{M 1}$. Because of its higher dielectric constant, $\mathrm{HfO}_{2}$ is used as the dielectric material next to the cavity region. Since the tunneling is taking place parallel to the channel in the proposed TFET biosensor; therefore, it is a point tunneling device.

\subsection{Simulation methodology and calibration}

Silvaco ATLAS tool is used for the numerical simulations of the proposed biosensor [19]. A non-local BTBT model is used to model the tunneling process accurately. Other models used

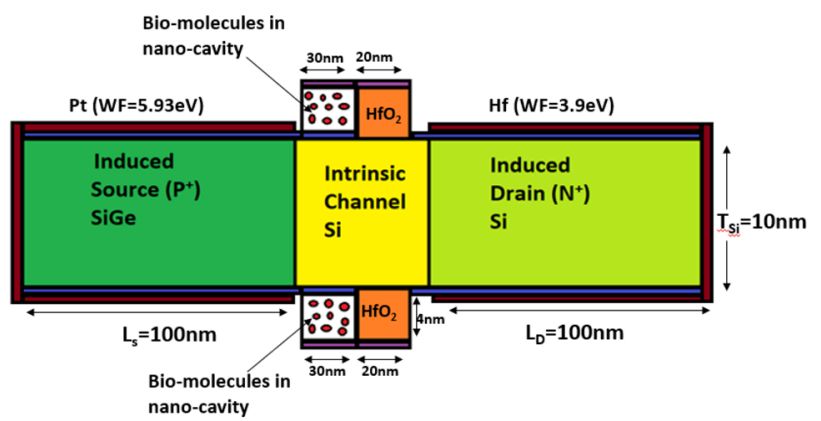

Fig. 1 Device structure of electrically doped dual metal gate SiGeheterojunction DG TFET biosensor 


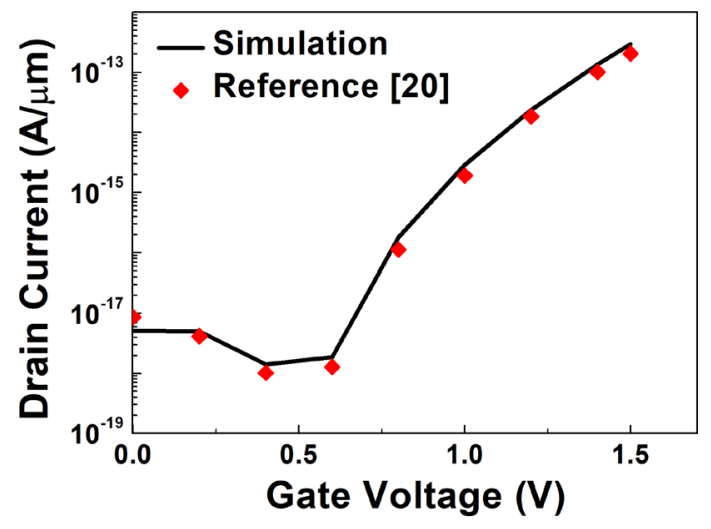

Fig. 2 Calibrated transfer characteristics of ED-DMG SiGe-heterojunction DG TFET biosensor with previously reported work in [20]

Table 1 Physical parameter specifications of proposed ED-DMG SiGe-heterojunction DG TFET biosensor

\begin{tabular}{ll}
\hline Parameter (symbol) & Value \\
\hline Gate length $\left(L_{g}\right)$ & $50 \mathrm{~nm}$ \\
Gate oxide thickness $\left(T_{\mathrm{ox}}\right)$ & $5 \mathrm{~nm}$ \\
Silicon thickness $\left(T_{\mathrm{si}}\right)$ & $10 \mathrm{~nm}$ \\
Cavity length $\left(L_{\text {cavity }}\right)$ & $30 \mathrm{~nm}$ \\
Cavity height $\left(T_{\text {cavity }}\right)$ & $3.5 \mathrm{~nm}$ \\
Source spacer $\left(L_{\mathrm{gs}}\right)$ & $3 \mathrm{~nm}$ \\
Drain spacer $\left(L_{\mathrm{gd}}\right)$ & $15 \mathrm{~nm}$ \\
S/D length $\left(L_{s} / L_{d}\right)$ & $100 \mathrm{~nm}$ \\
Gate workfunction $\left(\phi_{\mathrm{G}}(M 1 / M 2)\right)$ & $3.8 / 5.1 \mathrm{eV}$ \\
HfO2 length $\left(L_{\mathrm{HfO} 2}\right)$ & $20 \mathrm{~nm}$ \\
HfO2 height $\left(T_{\mathrm{HfO} 2}\right)$ & $4 \mathrm{~nm}$ \\
\hline
\end{tabular}

during the simulation are Shockley-Read-Hall (SRH) recombination model, Lombardi CVT model, Fermi-Dirac statistics, and field-dependent mobility model.

To validate the models and methods used, simulation results are calibrated against [20] which is shown in Fig. 2. For the calibration, carrier lifetimes $\left(\tau_{n}, \tau_{p}\right)$ are set to $10^{-1} \mu \mathrm{s}$, and band-to band tunneling parameters (me.tunnel, mh.tunnel) are set to 0.14 and 0.549 , respectively. Thermal velocity for electrons and holes $\left(V_{n}, V_{p}\right)$ are set as $1.08 \times 10^{7} \mathrm{~cm} / \mathrm{s}$ and $1.3 \times 10^{7}$ $\mathrm{cm} / \mathrm{s}$, respectively, while the saturation velocities are equal, i.e., $V_{\text {satn }}=V_{\text {satp }}=1.03 \times 10^{7} \mathrm{~cm} / \mathrm{s}$. Density of states at room temperature $(300 \mathrm{~K})$ are taken as $N_{c}=2.8 \times 10^{19} \mathrm{~cm}^{-3}$ and $N_{v}=1.04 \times 10^{19} \mathrm{~cm}^{-3}$.

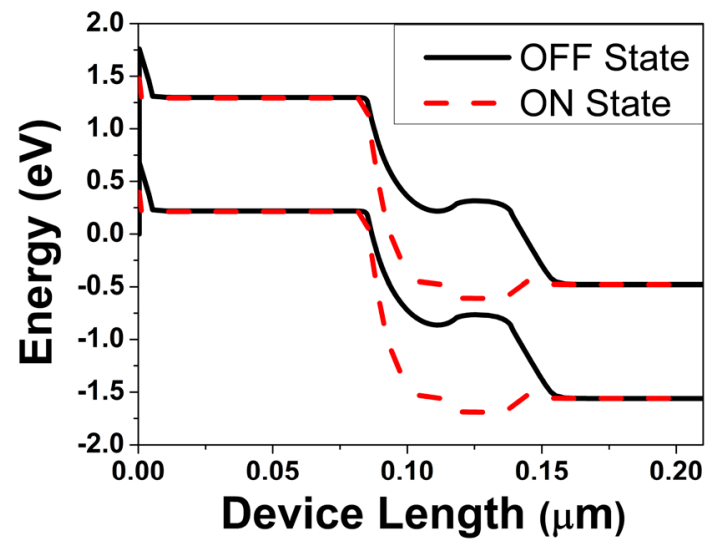

Fig. 3 Energy band diagram of the ED-DMG SiGe-heterojunction DG TFET biosensor in ON and OFF state
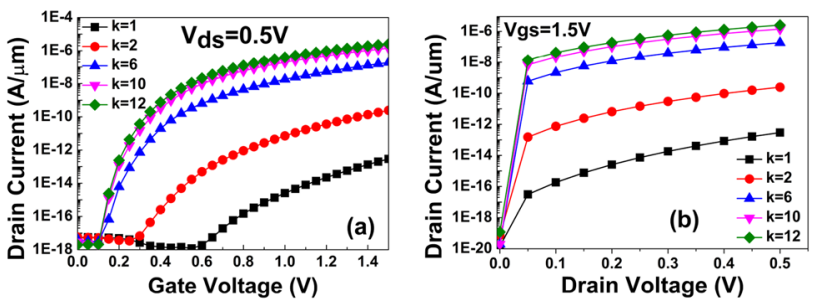

Fig. 4 Impact of variation in dielectric constant $(k)$ on a Transfer b Output characteristics of the ED-DMG SiGe-heterojunction DG TFET biosensor

\section{Results and discussion}

The energy band diagram of the proposed biosensor in OFF and ON state is shown in Fig. 3. When the device is in OFF state $\left(V_{\mathrm{gs}}=0 \mathrm{~V}, V_{\mathrm{ds}}=0.5 \mathrm{~V}\right)$, large tunneling barrier exists between valence band of source and conduction band of channel. This barrier reduces to a large extent when the device goes into ON-state $\left(V_{\mathrm{gs}}=1.5 \mathrm{~V}, V_{\mathrm{ds}}=0.5\right.$ $\mathrm{V})$ leading to current conduction through band to band tunneling (BTBT).

Transfer and output characteristics of the proposed biosensor for biomolecules having different dielectric constants in the cavity region are shown in Fig. 4. It is observed that upon functionalization of nanogap cavity by biomolecules, dielectric constant of the cavity increases. This scales down the effective oxide thickness (EOT) in accordance with IRDS standards. The reduced EOT provided by these high-k dielectric biomolecules, offers a high ON-current and makes it possible to sense the specific binding of biomolecules.

In most of the works reported on biosensor optimization, it is assumed that the cavity is fully and uniformly filled with biomolecules [18, 21-23]. In our previous work 
[24], we have also investigated the performance of a fully and uniformly filled cavity, i.e., ideal case. But this may not be the case in practical situations because the receptors or probes immobilize the biomolecules entering the cavity region. The hybridization process can stop before the cavity is completely packed. It prevents the entry of more biomolecules into the cavity region. A condition called steric hindrance is induced [25]. Therefore, extending our previous work, here we have studied the behaviour of partially filled cavity having fill factor less than $100 \%$ and different non-uniform profiles in the cavity region.

\subsection{Impact of fill factor on device performance}

For the completely filled cavity, the fill factor is $100 \%$, while the fill factor is less than $100 \%$ for the partially filled cavity. Figure 5 represents the cross-sectional view of fully and partially filled cavity regions. For fully filled cavity, length of cavity region is same as the length of biomolecules, i.e., $L_{\text {cavity }}=L_{\text {bio }}$. While for a partially filled cavity region, length of cavity region is more than the length of biomolecules, i.e., $L_{\text {cavity }}>L_{\text {bio }}$ leaving some portion of the cavity empty. Effective capacitance per unit area $\left(C_{\text {eff }}\right)$ is different for the fully filled and partially filled cavity regions as shown in Fig. 6 and $C_{\text {eff }}$ is calculated as given in Eqs. (4) to (7).

1. For uniformly and fully filled cavity, effective capacitance per unit area $\left(C_{\text {eff }}\right)$ of the cavity is

$$
C_{\text {eff }}=\frac{\epsilon_{\mathrm{SiO} 2} \times \epsilon_{\mathrm{Bio}}}{\left(\epsilon_{\mathrm{SiO} 2} \times t_{\mathrm{Bio}}\right)+\left(\epsilon_{\mathrm{Bio}} \times t_{\mathrm{SiO} 2}\right)}
$$

2. For uniformly and partially filled cavity, there are two different regions: one filled with biomolecules and other empty cavity region. Capacitance of the filled region is

$$
C_{\text {eff } 1}=\frac{\epsilon_{\mathrm{SiO} 2} \times \epsilon_{\mathrm{Bio}}}{\left(\epsilon_{\mathrm{SiO} 2} \times t_{\mathrm{Bio}}\right)+\left(\epsilon_{\mathrm{Bio}} \times t_{\mathrm{SiO} 2}\right)}
$$

, while capacitance for the empty region is

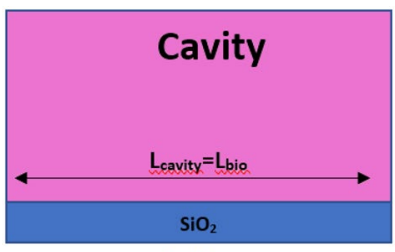

(a)

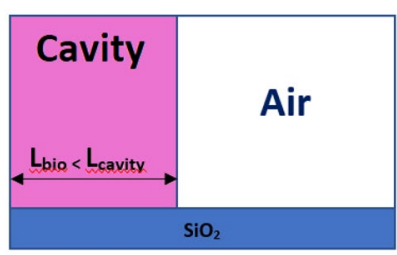

(b)
Fig. 5 a Cavity uniformly and fully filled with biomolecules b Cavity uniformly and partially filled with biomolecules

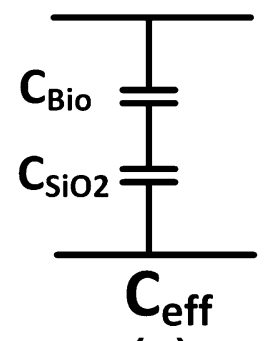

(a)

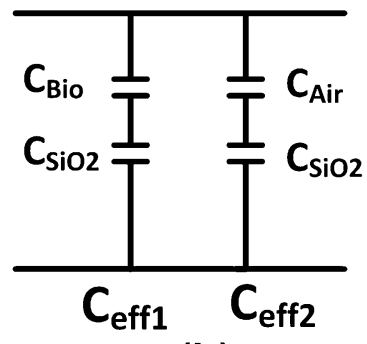

(b)
Fig. 6 Effectice capacitance per unit area for a completely filled cavity $\mathbf{b}$ partially filled cavity

$C_{\mathrm{eff} 2}=\frac{\epsilon_{\mathrm{SiO} 2} \times \epsilon_{\mathrm{Air}}}{\left(\epsilon_{\mathrm{SiO} 2} \times t_{\mathrm{Air}}\right)+\left(\epsilon_{\mathrm{Air}} \times t_{\mathrm{SiO} 2}\right)}$

Therefore, effective capacitance per unit area $\left(C_{\text {eff }}\right)$ of the cavity becomes

$C_{\text {eff }}=C_{\text {eff } 1}+C_{\text {eff2 }}$

where $\epsilon_{\mathrm{Bio}}, \epsilon_{\mathrm{SiO} 2}$ and $\epsilon_{\mathrm{Air}}$ is the permittivity of biomolecule, oxide layer and air gap, respectively, having thickness $t_{\mathrm{Bio}}, t_{\mathrm{SiO} 2}$ and $t_{\mathrm{Air}}$

To analyse the behaviour of an incomplete filled cavity, four different fill factors $(100 \%, 50 \%, 20 \%$, and $10 \%)$ are taken into consideration in this work. The transfer characteristics obtained for them are shown in Fig. 7. Maximum drain current is obtained for fully filled cavity, i.e., 100 $\%$ fill factor. The current remains almost same when the fill factor is reduced from 100 to $50 \%$. There is a small reduction in drain current when the fill factor is further reduced to $20 \%$, and a significant reduction in drain current is noticed for fill factor $10 \%$. This shows that there is a marginal variation in the drain current for a significant

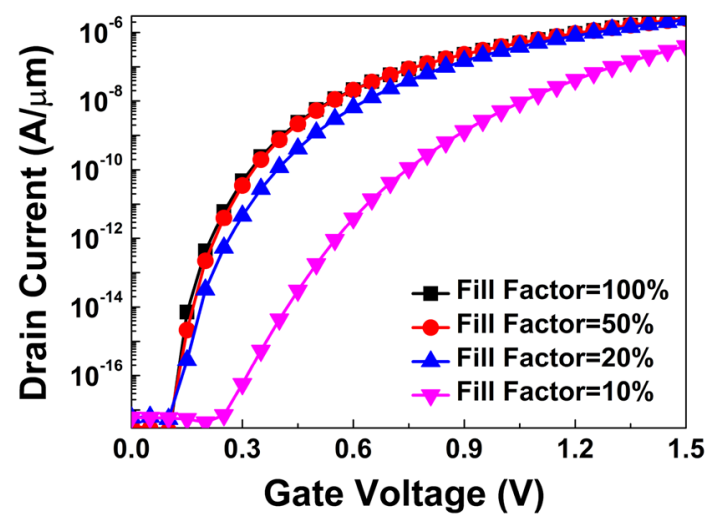

Fig. 7 Impact of variation in fill factor on transfer characteristics of ED-DMG SiGe-heterojunction DG TFET biosensor at $k=6$, $Q_{\text {Bio }}=0$ and $V_{\mathrm{ds}}=0.5 \mathrm{~V}$ 
variation in fill factor. Therefore, drain current does not have a very strong dependence on fill factor of cavity region.

\subsection{Impact of position of biomolecules inside the cavity on device performance}

Besides the fill factor, the position of biomolecules in the cavity region is also a very important parameter for consideration. Figure 8 shows the transfer characteristics for different positions of biomolecules inside the cavity having same fill factor. When fill factor is $50 \%$ and biomolecules are present at the source side, maximum drain current obtained is $10^{-6} \mathrm{~A} / \mu \mathrm{m}$, while for the same fill factor but biomolecules present at drain side, maximum drain current obtained is $10^{-13} \mathrm{~A} / \mu \mathrm{m}$. The reason being that when biomolecules are present at the source side they assist the tunneling process taking place at source-channel junction, and therefore, a higher value of drain current is obtained. A similar trend is obtained for $20 \%$ fill factor.

Figure 9a shows the energy band diagram for a half filled cavity having biomolecules at source and drain side. It is observed from the band diagram that tunneling width is wider when biomolecules are present at the drain side, i.e., $W 2>W 1$, which results in lesser current. Surface potential is also lower when biomolecules are present towards the drain side as shown in Fig. 9b. BTBT generation rate depends on the electric field at the tunneling junction. Higher is the electric field, better will be the BTBT generation rate and hence more tunneling current. Figure $9 \mathrm{c}$ shows the electric field is higher when biomolecules are accumulated at the source side thus, leading to an enhanced ON current for them.

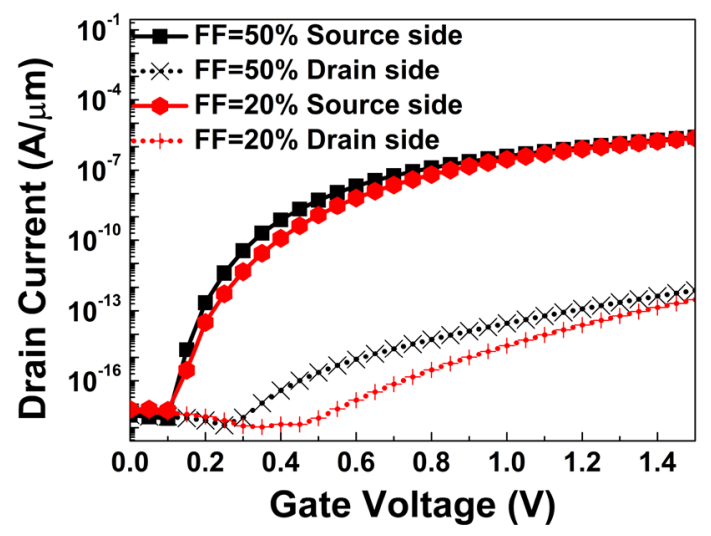

Fig. 8 Impact of position of biomolecules inside the cavity region on transfer characteristics of ED-DMG SiGe-heterojunction DG TFET biosensor at $k=6, Q_{\mathrm{Bio}}=0$ and $V_{\mathrm{ds}}=0.5 \mathrm{~V}$
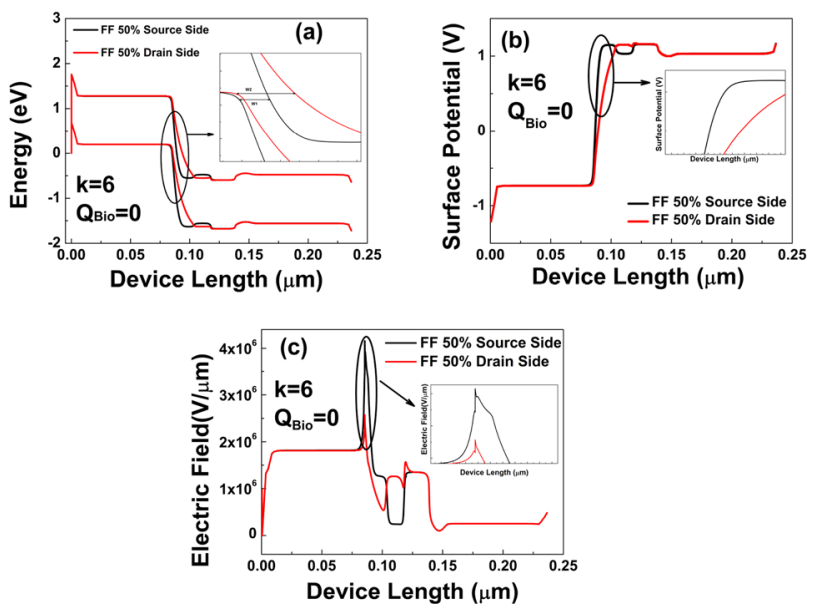

Fig. 9 Impact of position of biomolecules inside the cavity region on a Tunneling barrier width $\mathbf{b}$ Surface potential $\mathbf{c}$ Electric field at $k=6$, $Q_{\text {Bio }}=0$

\subsection{Impact of non-uniform profiles on device performance}

Uniform distribution of biomolecules in the nanocavity region is assumed in the previous sections. But the biomolecules can have non-uniform profiles because of steric hindrance. Four different non-uniform profiles (i) decreasing (ii) increasing (iii) concave and (iv) convex are considered here for analysis as shown in Fig. 10. The reason for choosing these non-uniform profiles is that they are easy to create, simulate and analyze and therefore, provide an overview of the behaviour of biosensor. To create different profiles, the cavity region is split into six sections where each section is equal in width $(5 \mathrm{~nm})$, while different heights of steps are chosen. For decreasing profile, height of steps is taken as $3.5 \mathrm{~nm}, 3 \mathrm{~nm}, 2.5 \mathrm{~nm}, 2 \mathrm{~nm}, 1.5 \mathrm{~nm}$, and $1 \mathrm{~nm}$ starting from the source channel junction, i.e., a difference of $0.5 \mathrm{~nm}$ among each step height. For increasing profile, opposite of this arrangement works. For concave profile, height of steps is taken as $3.5 \mathrm{~nm}, 3 \mathrm{~nm}, 2 \mathrm{~nm}, 2 \mathrm{~nm}, 3 \mathrm{~nm}, 3.5 \mathrm{~nm}$ and reverse of this arrangement works for the convex profile. The fill factor in each of the four cases is almost $64 \%$. Also, the biomolecules are charge neutral.

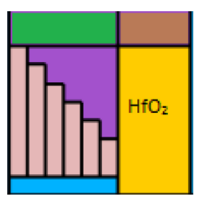

(a)

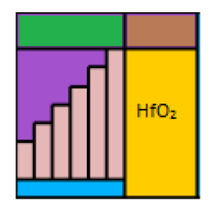

(b)

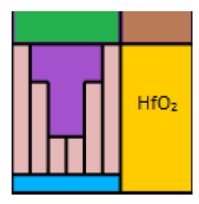

(c)

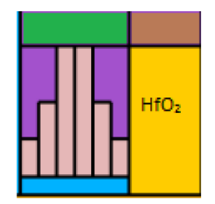

(d)
Fig. 10 Different non-uniform profiles in the cavity region a Decreasing $\mathbf{b}$ Increasing $\mathbf{c}$ Concave $\mathbf{d}$ Convex 


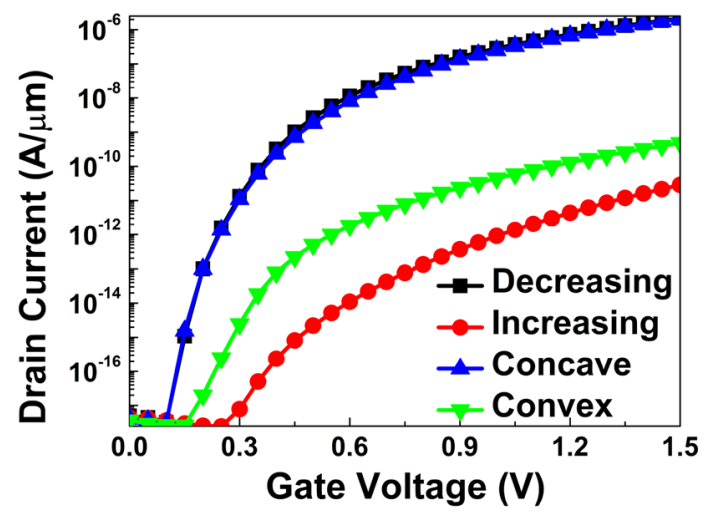

Fig. 11 Transfer characteristics of ED-DMG SiGe-heterojunction DG TFET biosensor for different non-uniform profiles at $k=6, Q_{\mathrm{Bio}}=0$ and $V_{\mathrm{ds}}=0.5 \mathrm{~V}$

Figure 11 shows the transfer characteristics for the non-uniform profiles considered. It is observed that maximum drain current is obtained for decreasing profile because of the maximum concentration of biomolecules near the source channel junction. The concentration decreases as we move towards the cavity end. Almost a similar response is obtained for concave profile because it again has the highest biomolecule concentration at the tunneling junction and lowest at the center of the cavity. Drain current for convex profile is lesser because it has maximum biomolecule concentration at the centre of the cavity. Minimum current is obtained for increasing profile because of lowest biomolecule concentration at tunneling junction which then increases towards the end of cavity.

\subsection{Impact of germanium molar fraction on device performance}

In the proposed biosensor structure, $\mathrm{Si}_{1-x} \mathrm{Ge}_{x}$ is used as the source material because of its lower band gap in comparison with $\mathrm{Si}$ which helps in improving the BTBT process at sourcechannel junction. Varying the germanium molar fraction will change the composition of the low bandgap material SiGe and this will lead to a change in the drain current of the device as depicted in Fig. 12. Four different molar fractions of Ge, i.e., $0.3,0.4,0.5$ and 0.6 are considered. It is observed from the results obtained that as the Ge molar fraction increases from 0.3 to 0.6 , drain current shows an improvement as shown in Table 2, which indicates better tunneling at the source-channel junction.
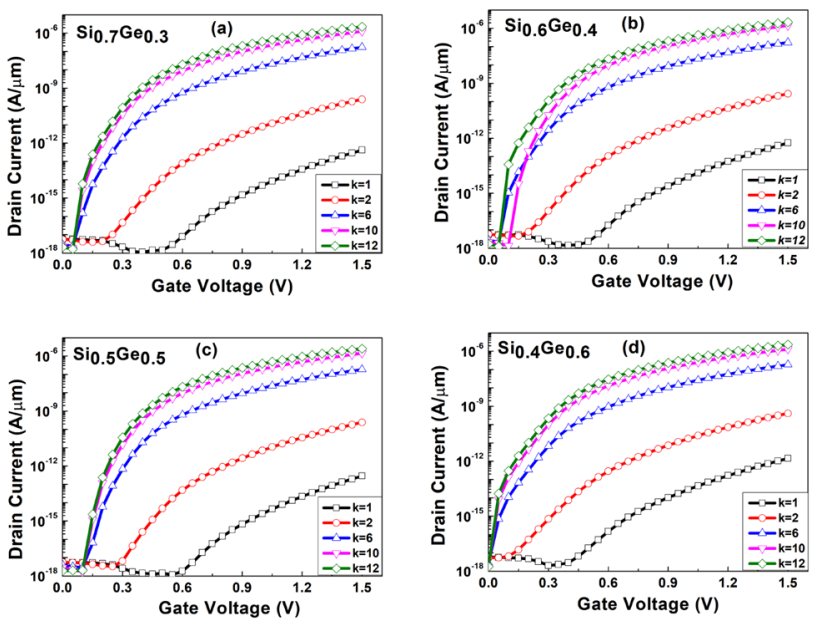

Fig. 12 Transfer characteristics of ED-DMG SiGe-heterojunction DG TFET biosensor for different Ge molar concentration at $V_{\mathrm{ds}}=0.5 \mathrm{~V}$ and $Q_{\text {Bio }}=0$

\section{Sensitivity analysis}

Sensitivity is a very important factor to aim at when developing a biosensor. Researchers are working towards designing highly sensitive biosensors that are capable of detecting even the smallest concentration of biomolecules present.

\subsection{Drain current sensitivity}

Drain current sensitivity $\left(S_{\text {Ids }}\right)$ of a biosensor is calculated as [26]

$S_{\text {Ids }}=\left(\frac{I_{D, \text { Bio }}-I_{D, \text { Air }}}{I_{D, \text { Air }}}\right)$

where $I_{D \text {,Bio }}$, and $I_{D \text {,Air }}$ are the drain currents with and without biomolecules, respectively. The variation in drain current sensitivity with \% fill factor is shown in Fig. 13. Maximum $S_{\text {Ids }}$ for fill factors $20 \%, 50 \%$ and $100 \%$ is almost same of the order of $10^{10}$ while for fill factor $10 \%, S_{\text {Ids }}$ is of the order of $10^{6}$.

Table 2 Maximum drain current obtained for different Ge molar fraction

\begin{tabular}{lllll}
\hline$k$ & \multicolumn{4}{l}{ Maximum drain current $(\mathrm{A} / \mu \mathrm{m})$} \\
\cline { 2 - 5 } & $\mathrm{Si}_{0.7} \mathrm{Ge}_{0.3}$ & $\mathrm{Si}_{0.6} \mathrm{Ge}_{0.4}$ & $\mathrm{Si}_{0.5} \mathrm{Ge}_{0.5}$ & $\mathrm{Si}_{0.4} \mathrm{Ge}_{0.6}$ \\
\hline 1 & $4.20 \times 10^{-13}$ & $5.66 \times 10^{-13}$ & $2.89 \times 10^{-13}$ & $1.43 \times 10^{-12}$ \\
2 & $2.48 \times 10^{-10}$ & $2.72 \times 10^{-10}$ & $2.44 \times 10^{-10}$ & $4.08 \times 10^{-10}$ \\
6 & $1.71 \times 10^{-7}$ & $1.70 \times 10^{-7}$ & $1.86 \times 10^{-7}$ & $1.88 \times 10^{-7}$ \\
10 & $1.23 \times 10^{-6}$ & $1.29 \times 10^{-6}$ & $1.30 \times 10^{-6}$ & $1.42 \times 10^{-6}$ \\
12 & $2.25 \times 10^{-6}$ & $2.19 \times 10^{-6}$ & $2.33 \times 10^{-6}$ & $2.55 \times 10^{-6}$ \\
\hline
\end{tabular}




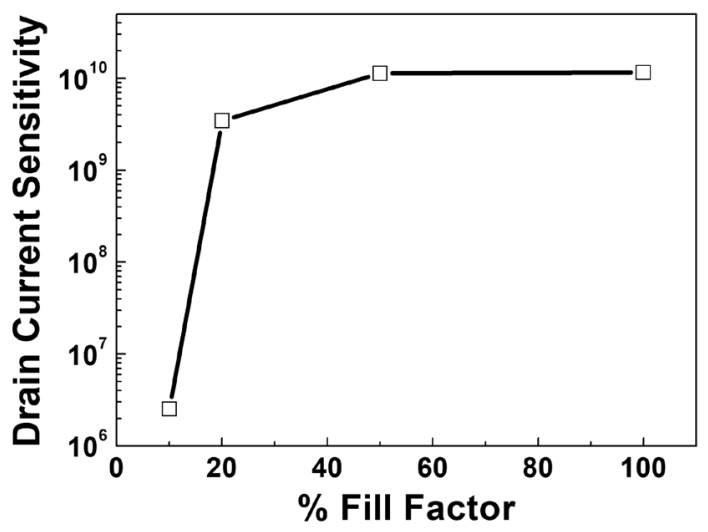

Fig. 13 Impact of variation in fill factor on drain current sensitivity for $k=6$, at $V_{\mathrm{gs}}=1.5 \mathrm{~V}$ and $V_{\mathrm{ds}}=0.5 \mathrm{~V}$

Table 3 Comparitive analysis of drain current sensitivity for different proposed works in the literature

\begin{tabular}{lll}
\hline References & Year & Approx. Sensitivity \\
\hline Proposed work & 2021 & $1.16 \times 10^{10}$ \\
Noor et al. [27] & 2017 & $4.82 \times 10^{7}$ \\
Sing et al. [22] & 2016 & $9.48 \times 10^{5}$ \\
Narang et al. [25] & 2015 & $3 \times 10^{6}$ \\
kanungo et al. [21] & 2015 & $1 \times 10^{6}$ \\
Srakar et al. [28] & 2012 & $9 \times 10^{3}$ \\
Hyungsoon et al. [29] & 2007 & $3 \times 10^{4}$ \\
\hline
\end{tabular}

\begin{tabular}{|c|c|c|c|c|c|}
\hline \multirow{2}{*}{$\begin{array}{c}\text { Cavity } \\
\text { divided in } \\
\text { six equal } \\
\text { parts }\end{array}$} & \multirow{2}{*}{$\begin{array}{l}\text { Shaded area } \\
\text { shows presence } \\
\text { of biomolecules }\end{array}$} & \multirow{2}{*}{$\begin{array}{l}\text { Fill } \\
\text { factor }\end{array}$} & \multicolumn{3}{|c|}{ Sensitivity } \\
\hline & & & $K=6$ & $K=10$ & $\mathrm{~K}=12$ \\
\hline Case 1 & & $50 \%$ & $9.20 \times 10^{8}$ & $8.47 \times 10^{9}$ & $1.69 \times 10^{10}$ \\
\hline Case 2 & & $50 \%$ & $1.53 \times 10^{7}$ & $6.15 \times 10^{7}$ & $9.20 \times 10^{7}$ \\
\hline Case 3 & & $66.6 \%$ & $9.34 \times 10^{8}$ & $8.54 \times 10^{9}$ & $1.69 \times 10^{10}$ \\
\hline Case 4 & & $66.6 \%$ & $3.09 \times 10^{5}$ & $5.72 \times 10^{5}$ & $6.90 \times 10^{5}$ \\
\hline Case 5 & & $33.3 \%$ & $7.74 \times 10^{8}$ & $7.96 \times 10^{9}$ & $1.62 \times 10^{10}$ \\
\hline Case 6 & & $33.3 \%$ & $2.70 \times 10^{5}$ & $5.20 \times 10^{5}$ & $6.34 \times 10^{5}$ \\
\hline
\end{tabular}

Fig. 14 Different cases showing the impact of position of biomolecules inside the cavity region on drain current sensitivity

Table 3 compares the approximate drain current sensitivity of previous reported works on biosensors. The proposed charge-plasma-based $\mathrm{SiGe}$ source biosensor has a maximum sensitivity of $1.13 \times 10^{10}$ for $k=12$ when the cavity is fully and uniformly filled with biomolecules which is better than the previously reported works.

Figure. 14 shows six cases having different locations of biomolecules inside the cavity region along with their

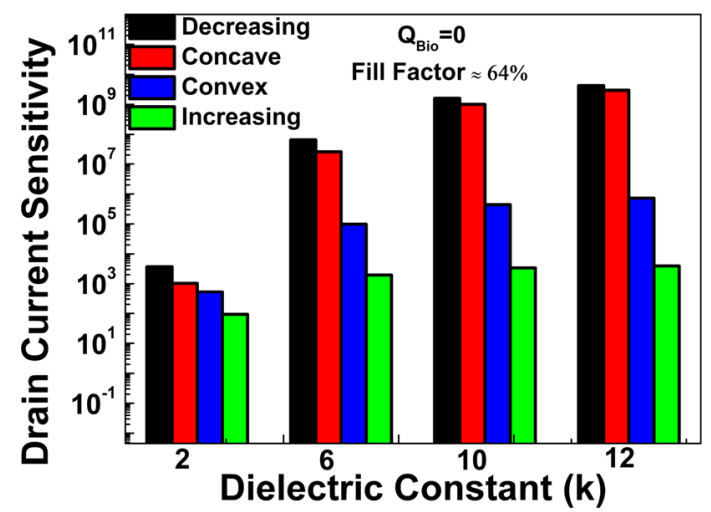

Fig. 15 Impact of variation in dielectric constant $(k)$ on drain current sensitivity for different non-uniform profiles at $V_{\mathrm{gs}}=1.5 \mathrm{~V}$ and $V_{\mathrm{ds}}=0.5 \mathrm{~V}$
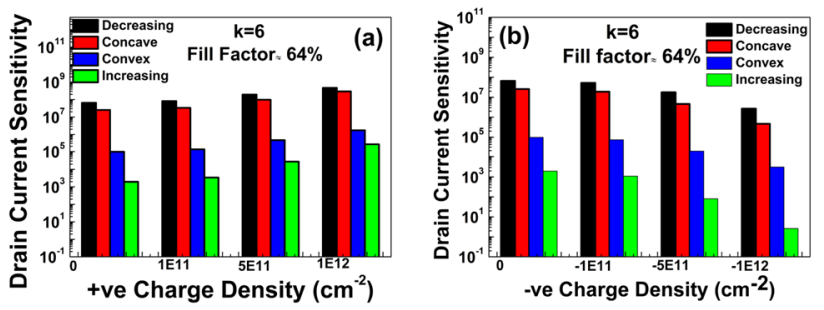

Fig. 16 Impact of variation in $\mathbf{a}+$ ve charge density $\mathbf{b}-$ ve charge density on drain current sensitivity for different non-uniform profiles at $V_{\mathrm{gs}}=1.5 \mathrm{~V}$ and $V_{\mathrm{ds}}=0.5 \mathrm{~V}$

drain current sensitivities. Shaded region shows the presence of biomolecules $(k>1)$, while the unshaded region shows the presence of air $(k=1)$. In cases 1 and 2 , the cavity is half filled, i.e., fill factor is $50 \%$, but the sensitivity is more for case 1 because all the biomolecules are immobilized towards the tunneling junction. While in case 2 , biomolecules are away from the source channel junction leading to lower sensitivity. Likewise, for cases 3 and 4 , fill factor is same $66.6 \%$, but better sensitivity is obtained for case 3 because of position of biomolecules near tunneling junction. A similar trend is observed for cases 5 and 6 having fill factor $33.3 \%$.

The drain current sensitivity is found to increase with an increase in the dielectric constant as shown in Fig. 15. Also among different non-uniform profiles considered, maximum $S_{I d s}$ is obtained for decreasing profile because of the maximum drain current for decreasing profile as shown in Fig. 11 followed by concave, convex, and increasing profiles.

The variation of drain current sensitivity with positive and negative charge density of the biomolecules is shown in Fig. 16. $S_{\text {Ids }}$ increases with an increase in positive charge density, while it shows an opposite trend in case of negative charge density [24]. 
Table 4 Drain current sensitivity values for neutral, positively charged and negatively charged biomolecules for different nonuniform profiles

\begin{tabular}{|c|c|c|c|c|c|}
\hline \multirow[t]{2}{*}{ Biomolecules in cavity } & \multirow[t]{2}{*}{ Dielectric constant } & \multicolumn{4}{|c|}{ Drain current sensitivity } \\
\hline & & Decreasing & Increasing & Convex & Concave \\
\hline \multirow[t]{4}{*}{ Neutral } & $k=2$ & $3.69 \times 10^{3}$ & $9.23 \times 10^{1}$ & $5.27 \times 10^{2}$ & $1.03 \times 10^{3}$ \\
\hline & $k=6$ & $6.56 \times 10^{7}$ & $1.95 \times 10^{3}$ & $9.71 \times 10^{4}$ & $2.54 \times 10^{7}$ \\
\hline & $k=10$ & $1.59 \times 10^{9}$ & $3.37 \times 10^{3}$ & $4.39 \times 10^{5}$ & $1.00 \times 10^{9}$ \\
\hline & $k=12$ & $4.23 \times 10^{9}$ & $3.90 \times 10^{3}$ & $7.24 \times 10^{5}$ & $2.94 \times 10^{9}$ \\
\hline \multirow[t]{4}{*}{ Positively charged } & $k=6, Q=0 \mathrm{C} / \mathrm{cm}^{2}$ & $6.55 \times 10^{7}$ & $1.95 \times 10^{3}$ & $9.71 \times 10^{4}$ & $2.54 \times 10^{7}$ \\
\hline & $k=6, Q=1 \mathrm{e} 11 \mathrm{C} / \mathrm{cm}^{2}$ & $8.16 \times 10^{7}$ & $3.39 \times 10^{3}$ & $1.34 \times 10^{5}$ & $3.37 \times 10^{7}$ \\
\hline & $k=6, Q=5 \mathrm{e} 11 \mathrm{C} / \mathrm{cm}^{2}$ & $1.93 \times 10^{8}$ & $2.78 \times 10^{4}$ & $4.47 \times 10^{5}$ & $9.71 \times 10^{7}$ \\
\hline & $k=6, Q=1 \mathrm{e} 12 \mathrm{C} / \mathrm{cm}^{2}$ & $4.69 \times 10^{8}$ & $2.61 \times 10^{5}$ & $1.75 \times 10^{6}$ & $2.92 \times 10^{8}$ \\
\hline \multirow[t]{4}{*}{ Negatively charged } & $\mathrm{k}=6, \mathrm{Q}=0 \mathrm{C} / \mathrm{cm}^{2}$ & $6.56 \times 10^{7}$ & $1.95 \times 10^{3}$ & $9.71 \times 10^{4}$ & $2.54 \times 10^{7}$ \\
\hline & $k=6, Q=-1 \mathrm{e} 11 \mathrm{C} / \mathrm{cm}^{2}$ & $5.21 \times 10^{7}$ & $1.09 \times 10^{3}$ & $6.97 \times 10^{4}$ & $1.88 \times 10^{7}$ \\
\hline & $k=6, Q=-5 \mathrm{e} 11 \mathrm{C} / \mathrm{cm}^{2}$ & $1.76 \times 10^{7}$ & $8.06 \times 10^{1}$ & $1.89 \times 10^{4}$ & $4.54 \times 10^{6}$ \\
\hline & $k=6, Q=-1 \mathrm{e} 12 \mathrm{C} / \mathrm{cm}^{2}$ & $2.66 \times 10^{6}$ & 2.61 & $3.13 \times 10^{3}$ & $4.62 \times 10^{5}$ \\
\hline
\end{tabular}

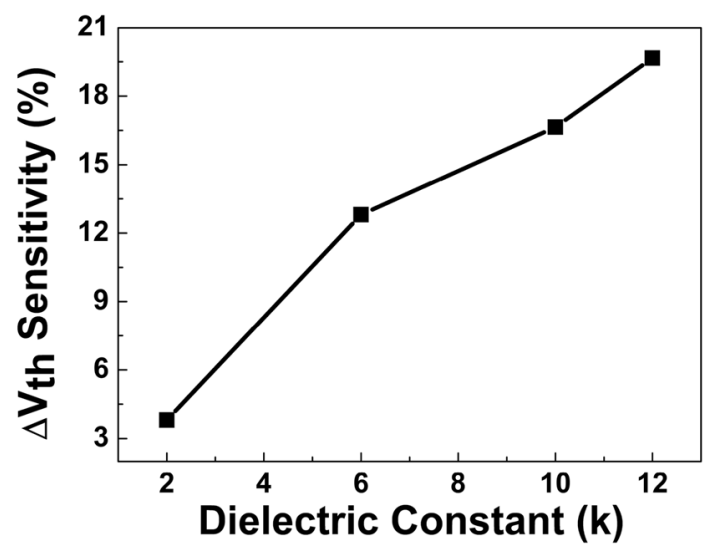

Fig. 17 Impact of variation in dielectric constant on Vth sensitivity for fully filled cavity at $V_{\mathrm{gs}}=1.5 \mathrm{~V}$ and $V_{\mathrm{ds}}=0.5 \mathrm{~V}$

The values of drain current sensitivity for neutral, positively charged and negatively charged biomolecules considering different non-uniform profiles are presented in Table 4.

\subsection{Threshold voltage sensitivity}

Change in threshold voltage is chosen as a sensing metric because threshold voltage varies with variation in dielectric constant and charge density of the biomolecules trapped in the cavity region. Threshold voltage sensitivity is calculated as [30]

$S_{V \text { th }}=\left(\frac{V_{\text {th,Air }}-V_{\text {th,Bio }}}{V_{\text {th,Air }}}\right)$

When the dielectric constant is increased from $k=1$ to $k=12$, overall threshold voltage of the biosensor decreases.
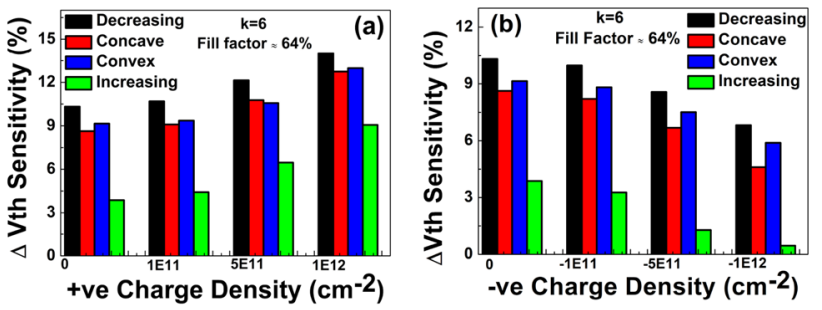

Fig. 18 Impact of variation in $\mathbf{a}+$ ve charge density $\mathbf{b}-\mathrm{ve}$ charge density on $V$ th sensitivity for different non-uniform profiles at $V_{\mathrm{gs}}=1.5 \mathrm{~V}$ and $V_{\mathrm{ds}}=0.5 \mathrm{~V}$

This leads to an increase in $S_{V \text { th }}$ as shown in Fig. 17. $S_{V \text { th }}$ increases from $3.8 \%$ (for $k=2$ ) to $19.6 \%$ (for $k=12$ ). Figure 18 shows the variation of $S_{V \text { th }}$ with positive and negative charge density. With increase in +ve charge density from 0 to $1 \mathrm{e} 12$, Vth sensitivity increases, while for increase in -ve charge density, Vth sensitivity decreases. Maximum $S_{V \text { th }}$ is obtained for decreasing profile followed by concave, convex and increasing profiles.

\section{Conclusion}

In this work, an electrically doped, dual metal gate, SiGeheterojunction, double gate dielectric modulated TFET biosensor is proposed. The proposed biosensor has a cavity towards the source side and high-k dielectric $\mathrm{HfO}_{2}$ besides the cavity region. The absence of physical doping makes the proposed biosensor immune to random dopant fluctuations and also cost-effective. The impact of fill factor on device performance is analyzed by considering four different fill factors, $100 \%, 50 \%, 20 \%$ and $10 \%$. Besides fill factor, position of biomolecules inside the cavity region also plays 
a crucial role and it is found that closer the biomolecules towards the tunneling junction, better will be the sensitivity. Six different cases having different locations of biomolecules inside the cavity region are studied in this work and a comparison of their sensitivities is presented. Different non-uniform profiles inside the cavity region are also analyzed with the help of numerical simulations. It is observed that decreasing profile has maximum drain current because of highest concentration of biomolecules at the source channel junction and therefore highest sensitivity. It is followed by concave and convex profiles. Increasing profile has the lowest sensitivity. Both drain current sensitivity and threshold voltage sensitivity have been used as sensing metrics to analyze the performance of the proposed biosensor.

Acknowledgements The authors would like to thank head of the department, Department of Electronics and Communication Engineering, Malaviya National Institute of Technology Jaipur for providing necessary support for carrying out the simulation work.

Author Contributions The main conception of this work is the brainchild of Basudha Dewan (Author 1). The first draft of the manuscript was written by Basudha Dewan. Shalini Chaudhary (Author 2) provided the necessary support regarding simulation and data interpretation. Menka Yadav (Author 3) supervised the work and made important discussions and modifications to the final manuscript.

Funding The authors have not received any funding for this work.

Availability of Data and Materials There are no linked research data sets for this submission.

\section{Declarations}

Conflict of interests The authors declare no competing interests.

Ethics approval and consent to participate Informed consent was obtained from all individual participants included in the study.

Consent for publication Informed consent was obtained from all individual participants included in the study.

Disclosure of potential conflicts of interest The authors declare that they have no conflict of interest.

Research involving Human Participants and/or Animals All procedures performed in this study involving human participants were in accordance with the ethical standards of the institutional and/or national research committee and with the 1964 Helsinki declaration and its later amendments or comparable ethical standards.

Informed consent Informed consent was obtained from all individual participants included in the study.

\section{References}

1. B. Acharya, G.P. Mishra et al., IEEE Sens. J. 20(23), 13969 (2020)
2. S.P. Mohanty, E. Kougianos, IEEE Potentials 25(2), 35 (2006)

3. P. Mehrotra, J. Oral Biol. Craniofac. Res. 6(2), 153 (2016)

4. L.C. Clark Jr., C. Lyons, Ann. N. Y. Acad. Sci. 102(1), 29 (1962)

5. D. Soni, D. Sharma, S. Yadav, M. Aslam, N. Sharma, Superlattices Microstruct. 113, 97 (2018)

6. J.Y. Kim, J.H. Ahn, S.J. Choi, M. Im, S. Kim, J.P. Duarte, C.H. Kim, T.J. Park, S.Y. Lee, Y.K. Choi, IEEE Trans. Nanotechnol. 11(2), 390 (2011)

7. M. Curreli, R. Zhang, F.N. Ishikawa, H.K. Chang, R.J. Cote, C. Zhou, M.E. Thompson, IEEE Trans. Nanotechnol. 7(6), 651 (2008)

8. B.V. Chandan, K. Nigam, D. Sharma, Micro Nano Lett. 13(4), 452 (2018)

9. B. Ghosh, M.W. Akram, IEEE Electron Dev. Lett. 34(5), 584 (2013)

10. D. Sarkar, K. Banerjee, in 70th Device Research Conference (IEEE, 2012), pp. 83-84

11. R. Narang, K.S. Reddy, M. Saxena, R. Gupta, M. Gupta, IEEE Trans. Electron Dev. 59(10), 2809 (2012)

12. S. Kanungo, S. Chattopadhyay, P.S. Gupta, K. Sinha, H. Rahaman, IEEE Trans. Electron Dev. 63(6), 2589 (2016)

13. M. Yadav, A. Bulusu, S. Dasgupta, Microelectron. J. 44(12), 1251 (2013)

14. N. Shafi, C. Sahu, C. Periasamy, IEEE Sens. J. 20(9), 4749 (2020)

15. C. Sahu, J. Singh, IEEE Electron Dev. Lett. 35(3), 411 (2014)

16. M. Moore. International roadmap for devices and systems (irds ${ }^{\mathrm{TM}}$ ) edition (2020)

17. V.D. Wangkheirakpam, B. Bhowmick, P.D. Pukhrambam, IEEE Trans. Nanotechnol. 19, 156 (2020)

18. A. Singh, S.I. Amin, S. Anand, Silicon 12(10), 2301 (2020)

19. S. Int, C. Santa Clara, et al., Santa Clara, CA, USA (2014)

20. M.J. Kumar, S. Janardhanan, IEEE Trans. Electron Dev. 60(10), $3285(2013)$

21. S. Kanungo, S. Chattopadhyay, P.S. Gupta, H. Rahaman, IEEE Trans. Electron Dev. 62(3), 994 (2015)

22. D. Singh, S. Pandey, K. Nigam, D. Sharma, D.S. Yadav, P. Kondekar, IEEE Trans. Electron Dev. 64(1), 271 (2016)

23. S. Kumar, Y. Singh, B. Singh, P.K. Tiwari, IEEE Sens. J. 20(21), 12565 (2020)

24. B. Dewan, S. Chaudhary, M. Yadav, Silicon pp. 1-10 (2021)

25. R. Narang, M. Saxena, M. Gupta, IEEE Trans. Nanotechnol. 14(3), 427 (2015)

26. S. Anand, A. Singh, S.I. Amin, A.S. Thool, IEEE Sens. J. 19(12), 4369 (2019)

27. S.L. Noor, M.Z.R. Khan, in 2017 IEEE Region 10 Humanitarian Technology Conference (R10-HTC) (IEEE, 2017), pp. 345-348

28. D. Sarkar, K. Banerjee, Appl. Phys. Lett. 100(14), 143108 (2012)

29. H. Im, X.J. Huang, B. Gu, Y.K. Choi, Nat. Nanotechnol. 2(7), 430 (2007)

30. M. Verma, S. Tirkey, S. Yadav, D. Sharma, D.S. Yadav, IEEE Trans. Electron Dev. 64(9), 3841 (2017)

Publisher's Note Springer Nature remains neutral with regard to jurisdictional claims in published maps and institutional affiliations. 